\title{
Assessment of Irradiation Performance in the Jules Horowitz Reactor (JHR) using the CARMEN Measuring Device
}

\author{
David Blanchet ${ }^{1}$, Muriel Antony ${ }^{2}$, Hubert Carcreff ${ }^{3}$, Sébastien François ${ }^{4}$, Philippe Guimbal ${ }^{5}$, \\ Bernard Pouchin ${ }^{1}$ \\ ${ }^{1}$ CEA Cadarache, DES/IRESNE/DER, F-13108 Saint-Paul-lez-Durance, France \\ ${ }^{2}$ CEA Cadarache, DES/DIMP/DCET, F-13108 Saint-Paul lez-Durance, France \\ ${ }^{3}$ CEA Saclay, DES/ISAS/DM2S, F-91190 Gif-sur-Yvette, France \\ ${ }^{4}$ CEA Cadarache, DES/DIMP/DPED, F-13108 Saint-Paul-lez-Durance, France \\ ${ }^{5}$ CEA Saclay, DES/ISAS/DMN, F-91190 Gif-sur-Yvette, France \\ david.blanchet@cea.fr
}

\begin{abstract}
The development of the JHR experimental devices rely on the operational feedback from previous French material testing reactors (i.e. SILOE and OSIRIS). The experimental devices used for the irradiation of structural material were already facing technological limitations, in particular regarding the control of irradiation temperature and of the thermal gradients in the experimental samples, which is essential to ensure the quality of the experiments. Obtaining satisfactory thermal fields (in compliance with the setpoint and the homogeneity) is all the more difficult as the level of nuclear heating is higher in the JHR. This paper attempts to characterize the irradiation conditions in different experimental positions of the JHR and to compare them with the conditions and the empirical criteria of maximum acceptable temperature measured in OSIRIS. The study shows that the irradiation conditions obtained inside the experimental devices can sometimes be significantly different from the measured conditions using instrumentation devices. The interpretation of the experimental results and their transposition to other situations will always require a calculation versus measurement adjustment and the intensive use of computer simulation. However, despite all simulation and transposition efforts, the control of temperature conditions is not yet fully demonstrated and nothing will ultimately replace experimental validation.
\end{abstract}

Keywords -Material Test Reactor (MTR), differential calorimeter, experimental devices, Reactor Pressure Vessel (RPV).

\section{INTRODUCTION}

$\mathrm{T}$ HE Jules Horowitz Reactor (JHR) is a Material Test Reactor currently under construction at Cadarache. It will serve to conduct experiments for the qualification of materials under irradiation and to produce radioisotopes for medical applications [1][2]. The material testing experiments require accurate monitoring of the irradiation conditions in terms of neutron flux (level and spectrum) and nuclear heating. A key aspect is to regulate the temperature level and to get uniform temperature distributions (absence of gradients) in the material samples during the irradiation. These requirements ensure the quality of the experiments and therefore contribute to the value of the irradiation service to future JHR clients.

CARMEN is a multi-detector measuring device that will provide a comprehensive characterization of the radiation field in the experimental channels of the JHR. The measurements performed with this device can serve as a preliminary assessment of unperturbed irradiation conditions, before the introduction of the real experimental device at that location. The present study aims at reproducing the CARMEN measurements by simulation and connecting them to the irradiation conditions expected inside the MICA and the OCCITANE test devices - which are respectively devoted to incore and out-core experiments for the irradiation of steel materials.

Section 2 presents the MICA and the OCCITANE test devices for structural material irradiation in the JHR. Section 3 presents the CARMEN measuring device. Section 4 examines modelling aspects for nuclear heating measurements with a differential calorimeter. In section 5, the study evaluates the transfer function between the CARMEN measurements (unperturbed conditions) and the actual nuclear heating inside test devices (perturbed conditions). Finally, section 6 attempts to integrate the feedback from the IRMA test device operation in the OSIRIS reactor.

\section{THE EXPERIMENTAL DEVICES FOR THE IRRADIATION OF STRUCTURAL MATERIALS IN THE JHR REACTOR}

\section{A. The MICA test device}

The MICA test device provides irradiation capabilities to study the structural material aging at high dpa-rates. There are 
ten in-core positions available to host this device: seven small channels located inside the fuel elements, and three large channels that could receive either a large irradiation device or an additional fuel element (see Fig. 1). Design studies and development of these devices rely on accurate nuclear heating calculations thanks to the experimental validation programs conducted in zero power reactors [3].

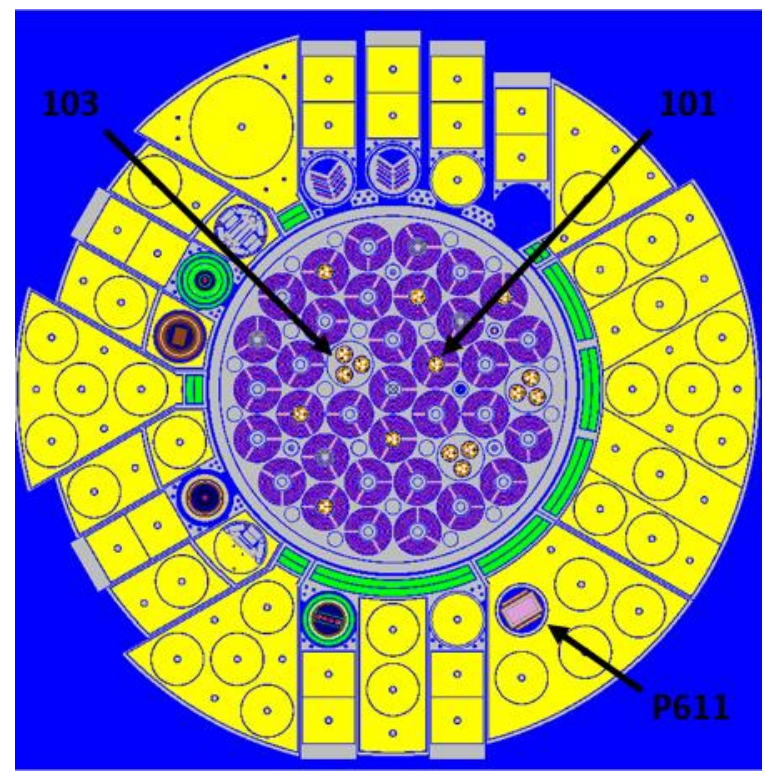

Fig. 1. Radial layout of the JHR reactor core model with TRIPOLI-4®.

As illustrated in Fig. 2, the MICA test device is inserted in the central axis of a fuel element. The material samples are loaded in a sample holder in the centre of the MICA device. The inner stainless steel tube of $24 \mathrm{~mm}$ in diameter limits the available space for the samples, which are immersed in static $\mathrm{NaK}$ eutectic. The good thermal conductivity of this liquid metal ensures optimal cooling and homogeneous thermal distribution in the samples. Surrounding the inner tube, some electric heating elements are embedded in order to reach and to regulate the setpoint temperature in the experimental zone. They allow flattening of the axial temperature gradients imposed by the axial nuclear heating profile. Several thermocouples are monitoring online temperatures while specific dosimeters are dedicated to evaluate, after irradiation, the integrated fast neutron fluence.
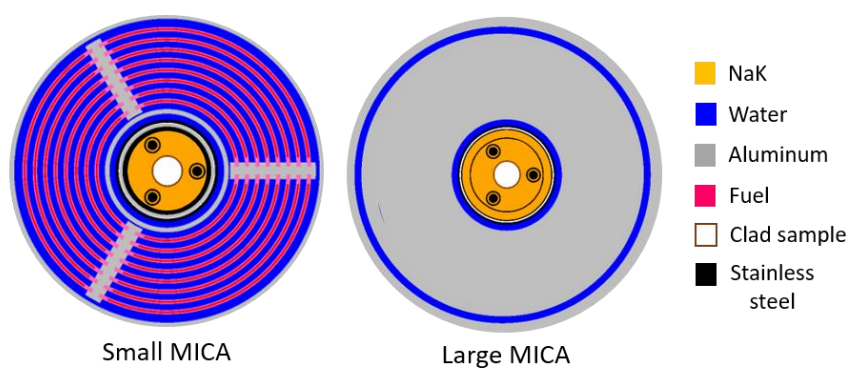

Fig. 2. The MICA test device.

\section{B. The OCCITANE test device}

The OCCITANE is a capsule-type experimental device used to study the accelerated aging and embrittlement of structural materials under irradiation, with the aim of anticipating the service life of steel vessels in Light Water Reactors. The RPV (Reactor Pressure Vessel) steel samples should be irradiated at low damage rate of about 1 mdpa per EFPD (Equivalent Full Power Day) to reach a total of 0.1 or 0.2 dpa (i.e. it takes about one year to reproduce the damage cumulated during 40 years in a standard NPP). Hence, the OCCITANE will be located outside the core, at position P611 (see Fig. 1), in the first row of JHR reflector. Independently of the neutron spectrum and nuclear heating imposed by the JHR, the test device must make it possible, if needed, to modify the irradiation conditions in the studied material samples. An important requirement is to be able to adapt the neutron spectrum and to regulate the internal temperature to cover the widest experimental field. Thus, the OCCITANE test device must meet the following specifications:

- A fast neutron flux in the range of $3 \cdot 10^{12} \leq \varphi>1 \mathrm{MeV} \leq 5 \cdot 10^{12} \mathrm{n} \cdot \mathrm{cm}^{-2} \cdot \mathrm{s}^{-1}$;

A homogeneous temperature distribution regulated between $260^{\circ} \mathrm{C}$ and $330^{\circ} \mathrm{C}$;

- A neutron spectrum ratio, denoted as Rs (see the forthcoming definition below), lying between $2.3 \leq \mathrm{Rs} \leq 2.5$.

The neutron spectrum transitions observed between internal and external walls of a RPV are often characterized by a physical quantity defined as the spectrum ratio: $\mathrm{Rs}=\Phi(\mathrm{E}>0.1 \mathrm{MeV}) / \Phi(\mathrm{E}>1 \mathrm{MeV})$, varying in the range of 2.4 at the inner wall, up to 8 at the outer wall [4]. In connection with experimental data from the irradiation programs previously carried out in the OSIRIS reactor, the test device must be able to reproduce a subset of irradiation conditions identical to those of the IRMA capsule in OSIRIS reflector, offering a spectrum ratio close to 2.4. However, it is also desirable to propose extended spectral tailoring capabilities, to be able to increase the spectrum ratio up to $\mathrm{Rs}=8$, if needed.

The experimental zone, covering a surface area of $30 \times 60 \mathrm{~mm}^{2}$, can host an arrangement of various type of samples (creep, tensile, Charpy and microstructure specimens) stacked on top of each other, on $60 \mathrm{~cm}$ height corresponding to the fissile column of the core. The axial damage gradient follows the fast neutron flux profile (see Fig. 5). Between each cycle, the sample holder is rotated by an angle of $180^{\circ}$ to azimuthally homogenise the neutron fluence in the experimental samples.

In order to control the irradiation temperature with homogeneous distribution, it is necessary to regulate with electric heating elements and to provide thermal insulation of the furnace, meanwhile reducing the nuclear heating in the experimental zone. Therefore, two screens are embedded in the device and optimized for that purpose (see Fig. 3):

A flat tungsten screen of variable thickness, placed against the sample holder, to attenuate the gamma rays coming directly from the core;

A cylindrical neutron screen of variable thickness attached to the outer refrigeration tube, made of boron carbide to absorb thermal neutrons. 
Thermal neutrons are indeed responsible for reactions of radiative capture in steel material, emitting gamma rays that contribute locally to nearly $30 \%$ of total nuclear heating in the experimental zone.

The inert (helium) gas gap provides a thermal insulation of the furnace. The heating elements are distributed along the experimental zone to compensate for the thermal gradients created by the axial and radial nuclear heating profile. In addition, the instrumentation includes typically 35 dosimeters installed as close as possible to the specimens to integrate the fast neutron fluence, and 18 thermocouples to provide in-line control of the temperatures inside the sample holder, and in-line regulation of the multi-zone heating system.

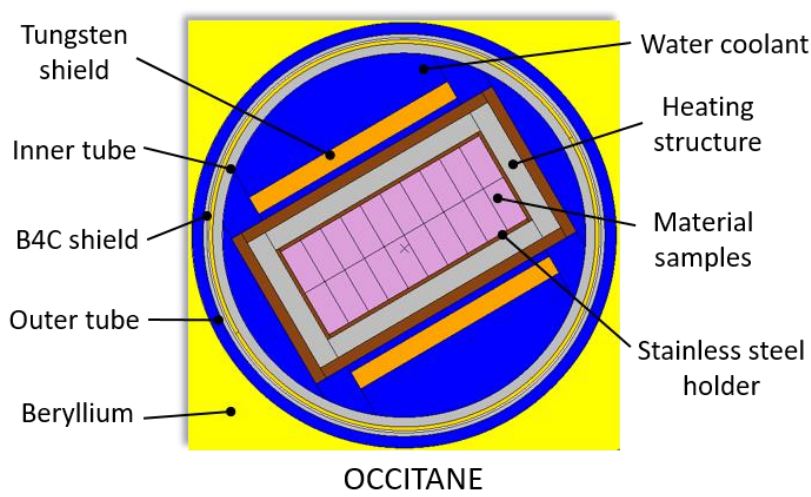

Fig. 3. The OCCITANE test device.

\section{THE CARMEN DEVICE}

The CARMEN is a multi-probe measuring device ensuring a high-level metrology service and providing a comprehensive nuclear radiation characterization in the experimental channels of the JHR. It will serve at first during reactor start-up commissioning tests, to verify core physics and safety parameters, and then, later, in support of reactor operation, to measure neutron flux and irradiation conditions. Hence, it will contribute to improve modelling aspects and better predict irradiation conditions for the different devices. It will also provide useful information for designing new equipment (in terms of performances or safety parameters).

The design of CARMEN integrates significant feedback from the experimental campaigns carried out in the OSIRIS reactor to qualify the instrumentation (CALMOS and CARMEN1) [5][6][7]. The qualification beyond $70 \mathrm{MW}$ up to the power level of the JHR $(100 \mathrm{MW})$ remains to be investigated. The system is equipped with state-of-the-art instrumentation, embedding 4 sensors (see Fig. 4):

(1) An ionization chamber (low sensitivity to neutrons) operating in current mode to measure the gamma flux;

(2) Two fission chambers, as part of the FNDS® system developed by the CEA [8], operating in fluctuation mode (practically insensitive to the gamma field) to measure the neutron flux: the first one equipped with a U235 deposit, providing high sensitivity to thermal neutrons, and the second one with a Pu242 deposit, being more selective in the fast energy range;
(3) A two cells differential calorimeter with an aluminumbased sample and a self-calibration system to measure the total heating rate.

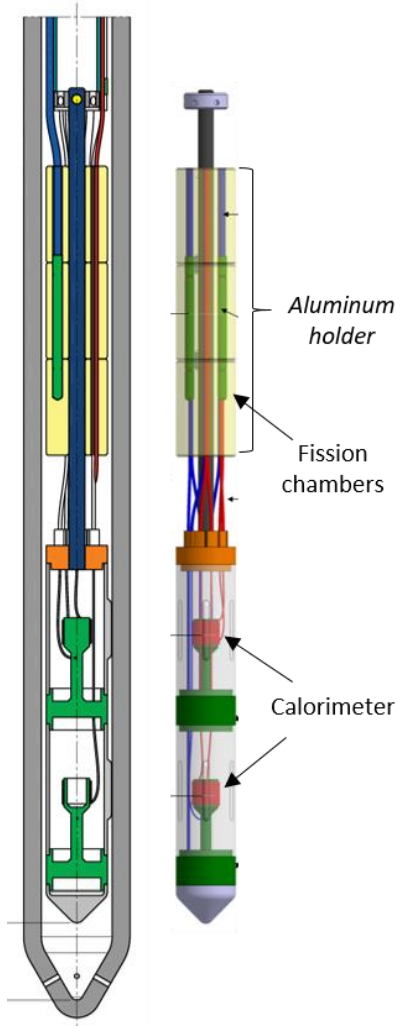

Fig. 4. The CARMEN device.

The use of various type of sensors having selective spectral responses to either neutron or gamma fields ensures better reliability and offers more possibilities of correlations for the interpretation and the transposition of the experimental results. CARMEN is well suited to access various experimental channels: on a fixed position in the core, and either on a fixed position or on a radial displacement system in the reflector. It has a motorized vertical displacement system with an incremental precision of $1 \mathrm{~mm}$, moving at a variable speed from 0.1 to $10 \mathrm{~mm} / \mathrm{s}$, between $-650 \mathrm{~mm}$ and $+1000 \mathrm{~mm}$ with respect to the axial core mid-plane (knowing that the fissile zone lies between $-300 \mathrm{~mm}$ and $+300 \mathrm{~mm}$ ). This wide axial displacement range allows the measurement cells to move up in a nearly zeroflux park position, which is of outmost importance for maintaining performance over time and avoiding drift problems, de-calibration, aging under flux (especially for fission chambers), or even damage of the sensors.

Fig. 5 depicts the axial profile of thermal $(\mathrm{E} \leq 0.625 \mathrm{eV})$ and fast $(\mathrm{E} \geq 1 \mathrm{MeV}$ ) neutron flux obtained at the MICA (in-core) and OCCITANE (reflector) irradiation positions. 


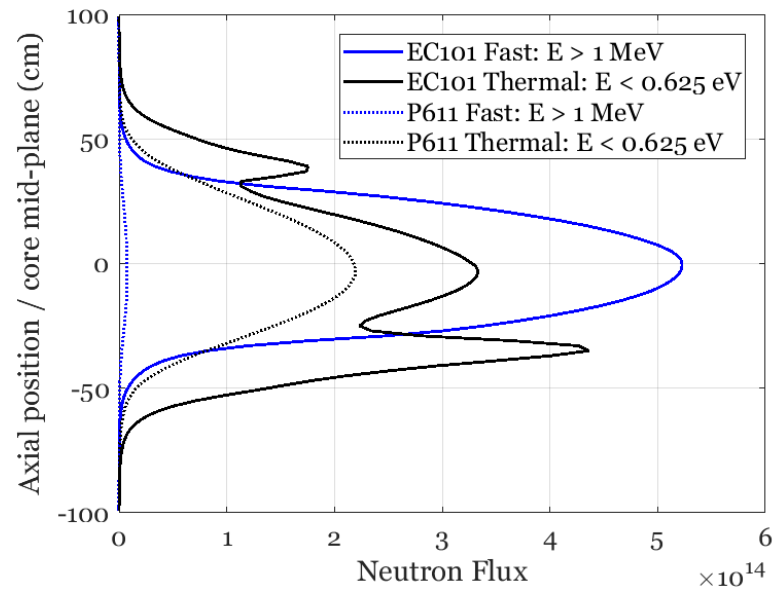

Fig. 5. Axial profile of thermal and fast neutron flux at the core (for MICA) and reflector (for OCCITANE) irradiation positions.

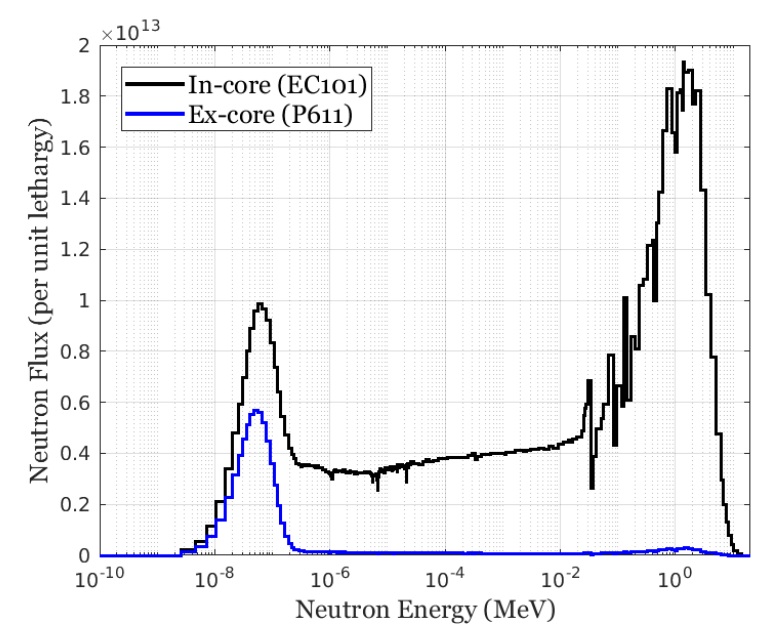

Fig. 6. Neutron spectrum distributions at the core and reflector irradiation positions.

\section{MODELLING OF NUCLEAR HEATING MEASURED BY CARMEN}

The JHR core neutronics is simulated in critical mode using the continuous-energy Monte-Carlo particle transport code TRIPOLI-4® [9], relying on the JEFF3.1 nuclear data library. Fig. 7 depicts the TRIPOLI-4® geometry of the aluminumbased differential calorimeter and, in Fig. 8, the axial profile of nuclear heating in aluminum obtained at the MICA and the OCCITANE irradiation positions. The advanced modelling of nuclear heating and energy deposition in the calorimeter requires the use of four-particle simulations involving neutron, photon, electron and positon. According to the results summarized in Table I and Table II, the impact of taking into account the transport of secondary charged particles produced by gamma interactions appears negligible. The absorbed dose obtained in $(n, \gamma)$ simulations is actually consistent with the absorbed dose obtained in (n, , e,p) simulations, which indicates that electronic equilibrium conditions are satisfied and that gamma heating is well-estimated by the use of a KERMA gamma response function.

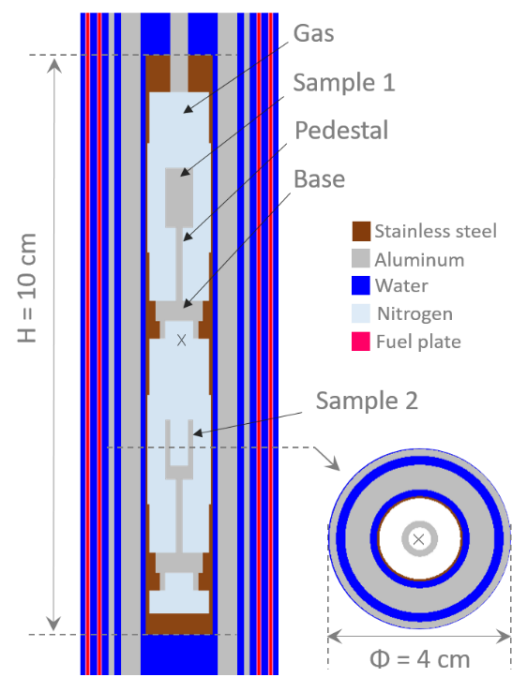

Fig. 7. TRIPOLI-4® geometry modelling of CARMEN's calorimeter.

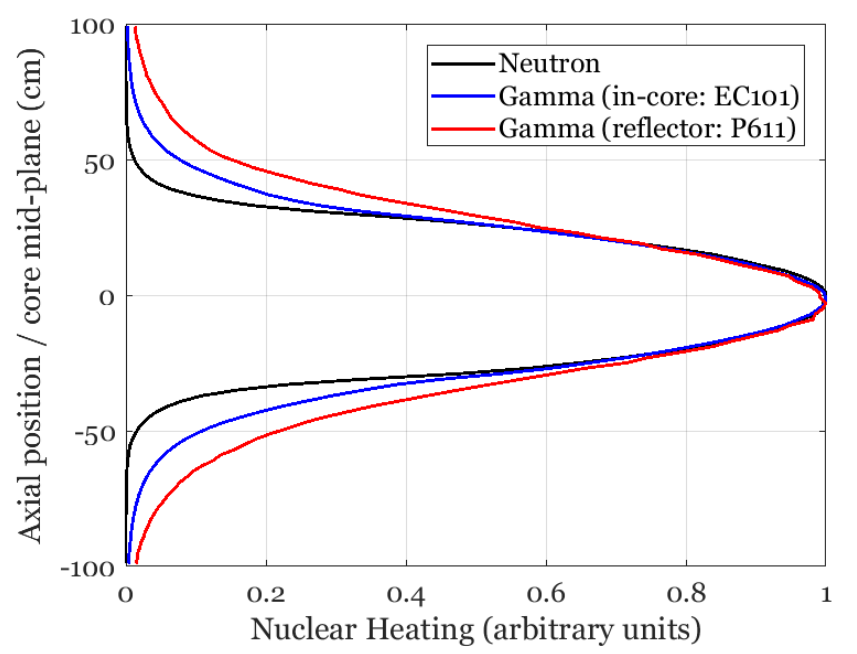

Fig. 8. Axial profile of nuclear heating (neutron and gamma) at the core (for MICA) and at the reflector (for OCCITANE) irradiation positions.

The operating principle of a differential calorimeter is to measure a temperature difference related to a difference in nuclear heating between an empty cell and a cell containing a material sample (see Fig. 7). Each cell is equipped with thermocouples (K-type) measuring a temperature difference proportional to the heat transfer by conduction through the pedestal, from the top ("hot side") down to the bottom ("cold side") part of the pedestal. Indeed, assuming a purely conductive heat transfer from the sample to the pedestal, neglecting the emissivity of inner surfaces (heat loss by radiation) and the heat conduction through the filling inert gas, and considering an optimal heat sink, the temperature difference is directly proportional to the difference in nuclear heating. The target temperature of the aluminum sample is around $300^{\circ} \mathrm{C}$ for a maximum nuclear heating of $\sim 20 \mathrm{~W} / \mathrm{g}$ expected in the JHR. 
TABLE I

TRIPOLI-4® $(\mathrm{N}, \gamma)$ AND $(\mathrm{N}, \curlyvee, \mathrm{P}, \mathrm{E})$ SIMULATIONS OF NUCLEAR HEATING MEASURED BY CARMEN'S CALORIMETER INSERTED IN JHR FUEL ELEMENT EC101, AT THE AXIAL MID-PLANE OF THE CORE.

\begin{tabular}{ccccc}
\hline \hline & $\begin{array}{c}\text { Mass } \\
\mathrm{g}\end{array}$ & $\begin{array}{c}\text { Total Heating } \\
(\mathrm{n}, \gamma) \pm 2 \sigma\end{array}$ & $\begin{array}{c}\text { Total Heating } \\
(\mathrm{n}, \mathrm{\gamma}, \mathrm{p}, \mathrm{e}) \pm 2 \sigma\end{array}$ & $\begin{array}{c}\text { Difference } \\
\% \pm 2 \sigma\end{array}$ \\
\hline \hline Cell 1 & 1.601 & $28.91 \mathrm{~W} \pm 0.2$ & $28.21 \mathrm{~W} \pm 0.3$ & $2.5 \% \pm 1.3 \%$ \\
\hline Cell 2 & 1.124 & $20.71 \mathrm{~W} \pm 0.1$ & $20.18 \mathrm{~W} \pm 0.3$ & $2.6 \% \pm 1.5 \%$ \\
\hline$\Delta=(1-2)$ & 0.477 & $8.20 \mathrm{~W} \pm 0.2$ & $8.04 \mathrm{~W} \pm 0.4$ & $2.1 \% \pm 5.8 \%$ \\
\hline$\Delta=(1-2)$ & 0.477 & $17.19 \mathrm{~W} / \mathrm{g} \pm 0.5$ & $16.84 \mathrm{~W} / \mathrm{g} \pm 0.9$ & $2.1 \% \pm 5.8 \%$ \\
\hline \hline
\end{tabular}

TABLE II

TRIPOLI-4® $(\mathrm{N}, \gamma)$ AND $(\mathrm{N}, \gamma, \mathrm{P}, \mathrm{E})$ SIMULATIONS OF NUCLEAR HEATING MEASURED BY CARMEN'S CALORIMETER INSERTED IN P611 POSITION, AT THE AXIAL MID-PLANE OF THE REFLECTOR

\begin{tabular}{ccccc}
\hline \hline & $\begin{array}{c}\text { Mass } \\
\mathrm{g}\end{array}$ & $\begin{array}{c}\text { Total Heating } \\
(\mathrm{n}, \gamma) \pm 2 \sigma\end{array}$ & $\begin{array}{c}\text { Total Heating } \\
(\mathrm{n}, \mathrm{\gamma}, \mathrm{p}, \mathrm{e}) \pm 2 \sigma\end{array}$ & $\begin{array}{c}\text { Difference } \\
\% \pm 2 \sigma\end{array}$ \\
\hline \hline Cell 1 & 1.601 & $2.53 \mathrm{~W} \pm 0.05$ & $2.37 \mathrm{~W} \pm 0.08$ & $6.8 \% \pm 4.2 \%$ \\
\hline Cell 2 & 1.124 & $1.82 \mathrm{~W} \pm 0.05$ & $1.68 \mathrm{~W} \pm 0.07$ & $7.9 \% \pm 4.8 \%$ \\
\hline$\Delta=(1-2)$ & 0.477 & $0.72 \mathrm{~W} \pm 0.07$ & $0.69 \mathrm{~W} \pm 0.11$ & $4.1 \% \pm 18.4 \%$ \\
\hline$\Delta=(1-2)$ & 0.477 & $1.50 \mathrm{~W} / \mathrm{g} \pm 0.15$ & $1.44 \mathrm{~W} / \mathrm{g} \pm 0.22$ & $4.1 \% \pm 18.4 \%$ \\
\hline \hline
\end{tabular}

According to Table I and Table II, the expected nuclear heating rate measured by CARMEN is $\sim 17 \mathrm{~W} / \mathrm{g}$ in the core, at the hottest irradiation position of the MICA test device, and $\sim 1.5 \mathrm{~W} / \mathrm{g}$ in the reflector, at the irradiation position of the OCCITANE test device.

Future work might address heat transfer modelling within the calorimeter, using a multi-physics code to get temperature distributions. This might be useful for having better insight into the CARMEN measurements interpretation and for the investigation of possible non-linearity effects that could be observed at high nuclear heating levels [10].

\section{DISCUSSION}

The assessment of irradiation conditions in various experimental positions in the Jules Horowitz Reactor core and reflector will rely on measurements performed using the CARMEN device. However, there is always a difference between the flux measured by sensors and the actual flux inside the experimental devices inserted at the same position. On the one hand, the introduction of the detector system may induce some perturbation of the environment that one wishes to characterize, and, on the other hand, the experimental device is modifying the external conditions to adapt them to irradiation specifications. Regarding the possible perturbations induced by the detector itself, design efforts were made for instance to limit the amount of moderator (water) close to the fission chambers while preserving margins for cooling requirements.

By design, the OCCITANE test device proposes spectral tailoring capabilities in compliance with the irradiation conditions specifically required for RPV steels. These conditions are achieved by adding screens of different natures to adjust the spectrum ratio and to reduce it at the target value of 2.4, whereas it is naturally close to $\sim 3$ in the sample holder when the device is located in the beryllium reflector.

Due to the lack of space available for adding screens in a small MICA test device inserted in a fuel element (only $24 \mathrm{~mm}$ in diameter), it is a far more difficult task to modify efficiently the spectrum ratio, which is by design naturally equal to 2.3 in the core. In spite of that lack of space, it is possible to add small neutron filters to decrease the part of thermal neutrons while preserving the fast flux level, with possible applications for Gen-IV material irradiations for instance. However, there is much more latitude in the large MICA test device occupying a whole in-core location (without fuel element), since there is up to $80 \mathrm{~mm}$ in diameter of free space available that could allow for a spectrum ratio adjustment, at least in the range of 2.0 up to 3.5 (see Table III). Therefore, in these situations, one can expect a difference between the spectral responses of detectors in unperturbed conditions and the actual spectral conditions adjusted by design in the experimental devices.

TABLE III

TAILORING CAPABILITIES IN A MICA DEVICE OCCUPYING A LARGE POSITION.

\begin{tabular}{|c|c|c|c|c|c|}
\hline \multicolumn{2}{|c|}{ Performance in Al Target } & \multicolumn{4}{|c|}{ Neutron Screen } \\
\hline Parameter & Units & $\mathrm{H}_{2} \mathrm{O}$ & $\mathrm{Al}$ & $\mathrm{Be}$ & $\mathrm{W}$ \\
\hline$\Phi_{\mathrm{E} \leq 0.625 \mathrm{eV}}$ & n.cm-2.s s $^{-1}$ & $1.6 \cdot 10^{15}$ & $2.8 \cdot 10^{14}$ & $4.9 \cdot 10^{14}$ & $1.3 \cdot 10^{14}$ \\
\hline$\Phi_{0.625 \mathrm{eV}} \leq \mathrm{E} \leq 0.1 \mathrm{MeV}$ & n.cm ${ }^{-2} \cdot s^{-1}$ & $6.7 \cdot 10^{14}$ & $8.2 \cdot 10^{14}$ & $1.1 \cdot 10^{14}$ & $4.7 \cdot 10^{14}$ \\
\hline$\Phi_{\mathrm{E} \geq 0.1 \mathrm{MeV}}$ & n.cm ${ }^{-2} \cdot \mathrm{s}^{-1}$ & $5.9 \cdot 10^{14}$ & $9.2 \cdot 10^{14}$ & $8.7 \cdot 10^{14}$ & $7.7 \cdot 10^{14}$ \\
\hline$\Phi_{\mathrm{E}} \geq 1 \mathrm{MeV}$ & $\mathrm{n} \cdot \mathrm{cm}^{-2} \cdot \mathrm{s}^{-1}$ & $2.9 \cdot 10^{14}$ & $3.9 \cdot 10^{14}$ & $3.4 \cdot 10^{14}$ & $2.2 \cdot 10^{14}$ \\
\hline$\Phi_{\geq 0.1 \mathrm{MeV}} / \Phi_{\geq 1 \mathrm{MeV}}$ & - & 2.0 & 2.3 & 2.5 & 3.5 \\
\hline Neutron Heating & $\mathrm{W} / \mathrm{g}$ & 0.4 & 0.6 & 0.5 & 0.4 \\
\hline Gamma Geating & $\mathrm{W} / \mathrm{g}$ & 17.0 & 11.7 & 13.4 & 2.8 \\
\hline Total Heating & $\mathrm{W} / \mathrm{g}$ & 17.4 & 12.3 & 13.9 & 3.2 \\
\hline
\end{tabular}

CARMEN's calorimeter has a large dynamic measurement range going from lower than $0.5 \mathrm{~W} / \mathrm{g}$ up to $20 \mathrm{~W} / \mathrm{g}$ (in aluminum). Measurements in the inner hole of fuel elements using the differential calorimeter are perfectly matching the nuclear heating conditions in the experimental samples of the MICA device (the transfer function remains the identity function). On the contrary, there is a significant difference between the nuclear heating in the OCCITANE material samples $(\sim 0.5 \mathrm{~W} / \mathrm{g})$ and the measurements performed using CARMEN at that same location $(\sim 1.5 \mathrm{~W} / \mathrm{g})$. In any case, the interpretation of experimental results by the calorimeter and their transposition to other situations will always require a calculation versus measurement adjustment and the intensive use of simulation.

\section{MODELLING OF A GRAPHITE-BASED DOUBLE- DIFFERENTIAL CALORIMETER IN OSIRIS}

The previous experimental program of RPV steel irradiations in OSIRIS was stressing the impossibility to ensure optimal temperature regulation in material samples (or specimens) when the measured nuclear heating by a graphite-based doubledifferential calorimeter was exceeding around $0.5 \mathrm{~W} / \mathrm{g}$. This empirical criterion was relying only on experimental characterizations performed with this calorimeter. The interpretation and the direct transposition of this criterion in JHR configuration is not an easy task. There are indeed several structural differences between OSIRIS and JHR core configurations, resulting in different irradiation conditions and finally each device has specific design features.

Fig. 9 and Fig. 10 present the radial layout of the OSIRIS reactor core and the IRMA capsule (containing the steel specimens for aging under irradiation) inserted in the water reflector. Fig. 11 describes the graphite-based calorimeter used to measure nuclear heating at the location of the IRMA capsule. 


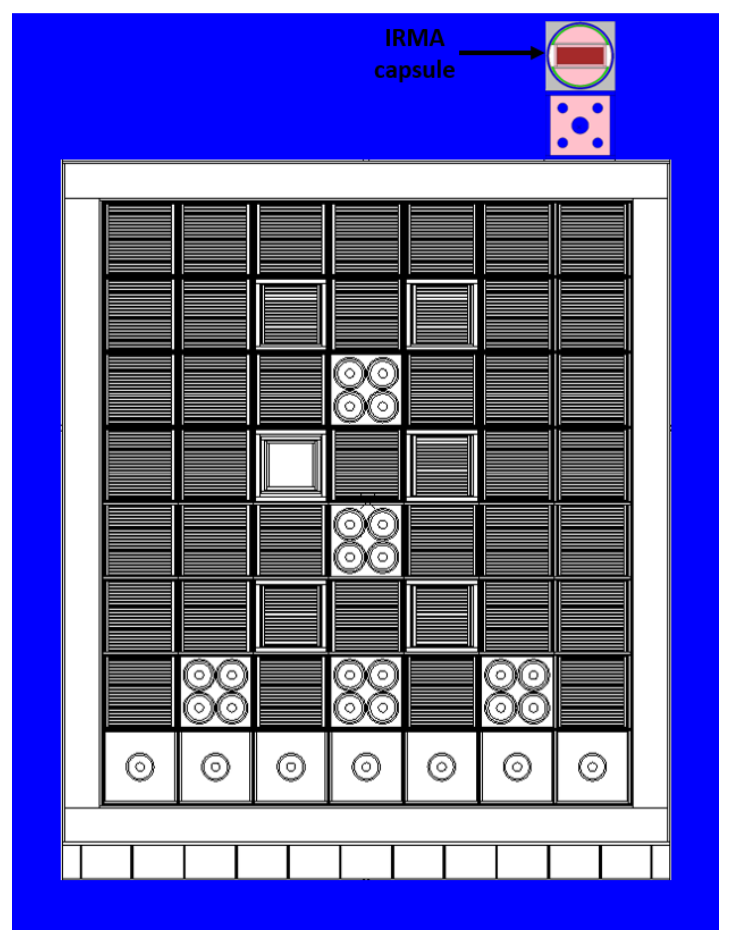

Fig. 9. Radial layout of the OSIRIS reactor core modelling with TRIPOLI-4®

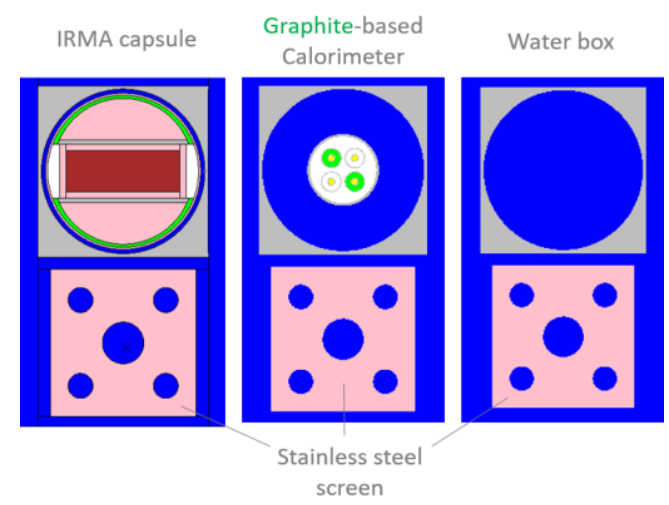

Fig. 10. TRIPOLI-4® modelling of the IRMA capsule and the graphite-based double differential dosimeter in the water reflector of the OSIRIS MTR.

Table IV presents results of energy deposition in the calorimeter, obtained by Monte-Carlo simulations with the TRIPOLI-4® code. It seems that there is no significant difference induced by the transport of secondary charged particles. Although these results neglect the contribution of delayed gamma emissions (produced by decay of fission products and activation products), they provide a first estimate of the nuclear heating measured by the calorimeter $(\sim 0.4 \mathrm{~W} / \mathrm{g})$, and a relative comparison with the nuclear heating in the experimental zone (sample holder) of the IRMA capsule, evaluated at $\sim 0.3 \mathrm{~W} / \mathrm{g}$. Therefore, the previously mentioned criterion of $0.5 \mathrm{~W} / \mathrm{g}$ of maximum nuclear heating measured by the calorimeter was actually corresponding to $0.375 \mathrm{~W} / \mathrm{g}$ $(=0.5 * 0.3 / 0.4)$ in the experimental zone of the IRMA capsule. A direct transposition of this criterion to JHR configuration is not obvious and requires further analysis.

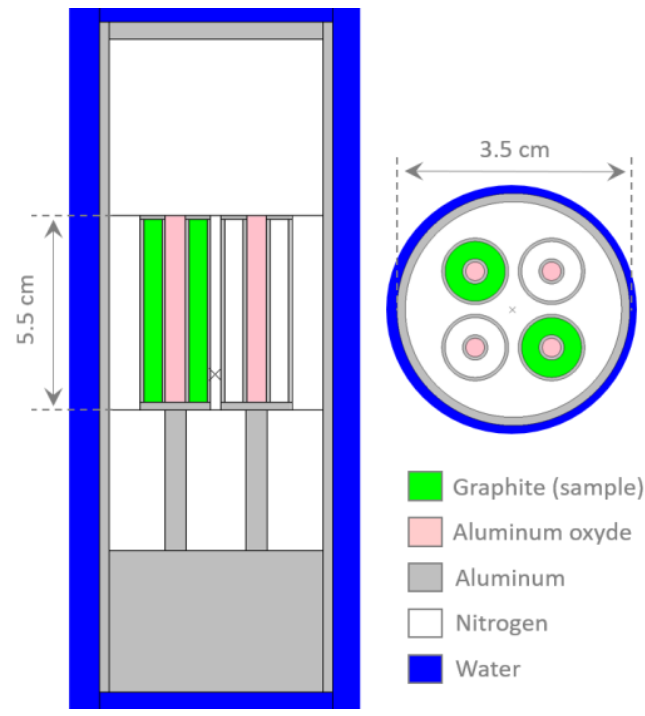

Fig. 11. Detailed geometry of the graphite-based double differential dosimeter.

TABLE IV

TRIPOLI-4® SIMULATIONS OF NUCLEAR HEATING (PROMPT GAMMA EMISSION ONLY) MEASURED BY A GRAPHITE-BASED DOUBLE-DIFFERENTIAL CALORIMETER INSERTED AT THE IRRADIATION POSITION OF IRMA TEST

\begin{tabular}{ccccc}
\multicolumn{5}{c}{ DEVICE IN OSIRIS. } \\
\hline \hline $\begin{array}{c}\text { Mass } \\
\mathrm{g}\end{array}$ & $\begin{array}{c}\text { Total Heating } \\
(\mathrm{n}, \mathrm{\gamma}) \pm 2 \sigma\end{array}$ & $\begin{array}{c}\text { Total Heating } \\
(\mathrm{n}, \mathrm{\gamma}, \mathrm{p}, \mathrm{e}) \pm 2 \sigma\end{array}$ & $\begin{array}{c}\text { Difference } \\
\% \pm 2 \sigma\end{array}$ \\
\hline \hline Cell 1 & 18.12 & $7.59 \mathrm{~W} \pm 0.11$ & $7.03 \mathrm{~W} \pm 0.08$ & $8.0 \% \pm 2.0 \%$ \\
\hline Cell 2 & 8.24 & $3.53 \mathrm{~W} \pm 0.07$ & $3.04 \mathrm{~W} \pm 0.05$ & $16.4 \% \pm 2.9 \%$ \\
\hline$\Delta=(1-2)$ & 9.89 & $4.06 \mathrm{~W} \pm 0.12$ & $3.99 \mathrm{~W} \pm 0.10$ & $1.6 \% \pm 4.1 \%$ \\
\hline$\Delta=(1-2)$ & 9.89 & $0.41 \mathrm{~W} / \mathrm{g} \pm 0.01$ & $0.40 \mathrm{~W} / \mathrm{g} \pm 0.01$ & $1.6 \% \pm 4.1 \%$ \\
\hline \hline
\end{tabular}

\section{CONCLUSION}

CARMEN is a multi-probe measurement device ensuring a high-level metrology service for the characterization of nuclear radiations in the experimental channels of the JHR. The present study evaluates the irradiation conditions as measured by CARMEN, and compare them to the actual conditions in two experimental devices, namely MICA and OCCITANE, for the irradiation of structural materials, respectively at high dpa rate in the core, and at low dpa rate in the reflector.

Apart from neutron spectrum aspects, the study focused on nuclear heating since it plays a major role in temperature regulation inside these two devices, which is of primary importance for the quality of future irradiation experiments. Advanced modelling of the energy deposition in the aluminum sample of the CARMEN calorimeter is performed using continuous-energy Monte-Carlo particle transport simulations with the TRIPOLI-4® code. These simulations exhibited the absence of transport effects from the secondary charged particles. The choice of aluminum as material sample for the calorimeter appears to be more representative of the JHR environment (many structures of the core are made of aluminum). In addition, aluminum is less sensitive to fast neutrons, in comparison to graphite.

The study evaluated also a transfer function between the CARMEN measurements and the actual irradiation conditions for structural materials inside test devices (MICA and OCCITANE). The interpretation of the experimental results 
and their transposition to other situations will always require a calculation versus measurement adjustment and the intensive use of computer simulation. An attempt was made to integrate the feedback from the IRMA capsule, which was dedicated to RPV steel irradiations in the OSIRIS reactor, and to transpose it for JHR. In particular, there are on-going investigations to analyse an empirical criterion on nuclear heating measured by a graphite-based double-differential calorimeter in unperturbed conditions, which was required not to exceed $0.5 \mathrm{~W} / \mathrm{g}$ in order to guarantee, when the IRMA capsule is in its location, the optimal temperature regulation in the capsule. The direct transposition of this criterion on the measured nuclear heating rate is not an easy task, taking into account all differences between core configurations, irradiation conditions and design of the devices. Preliminary comparisons do not yet demonstrate that temperature regulation is ensured for the OCCITANE device in the JHR. Therefore, it will be necessary to verify the thermal hydraulic characteristics beforehand, using dedicated model experiments for validation.

Future work will address heat transfer modelling within the calorimeter to get temperature distributions using a multi physics code. This might be useful for having better insight into the CARMEN measurements interpretation and for the investigation of possible non-linearity effects that could be observed at high nuclear heating levels.

\section{REFERENCES}

[1] J.P. Coulon et al., "Radioisotopes Production for Medical Use: Preliminary Design of the Jules Horowitz Reactor Facilities", RRFM IGORR, March 18-22 2012 Prague, Czech Republic.

[2] G. Bignan et al., "The Jules Horowitz Reactor: a new high performances Europeans MTR with modern experimental capacities: toward an international centre of excellence”, RRFM - IGORR, March 18-22 2012 Prague, Czech Republic.

[3] C. Vaglio-Gaudard et al., "Analysis of the AMMON experimental program in the EOLE facility supporting the qualification of the JHR neutron and photon tools", ANIMMA, 2013, pp. 1-9, DOI: 10.1109/ANIMMA.2013.6727887.

[4] Koji Fukuya, "Current understanding of radiation-induced degradation in light water reactor structural materials", Journal of Nuclear Science and Technology, 50:3, 213-254,DOI: 10.1080/00223131.2013.772448, (2013).

[5] H. Carcreff, L. Salmon, V. Lepeltier, Jm. Guyot, E. Bouard, "Last Improvements of the CALMOS Calorimeter Dedicated to Thermal Neutron Flux and Nuclear Heating Measurements inside the OSIRIS Reactor", ANIMMA, Jun 2017, Lieges, Belgium. 〈hal-02417753〉.

[6] Lyoussi et al., "Advanced methodology and instrumentation for accurate on line measurements of neutron, photon and nuclear heating parameters inside Jules Horowitz MTR reactor", in Proceedings of RRFM '12, Prague, Czech Republic, Mar. 2012.

[7] D. Fourmentel et al., "Nuclear Heating Measurements in Material Testing Reactor: A Comparison between a Differential Calorimeter and a Gamma Thermometer", IEEE Transactions on Nuclear Science, Vol. 60, ํ1, February 2013.

[8] L. Barbot, D. Fourmentel, J.-F. Villard, C. Destouches, B. Geslot et al., In-pile qualification of the fast-neutron-detection-system. EPJ Web of $\begin{array}{llll}\text { Conferences, } & \text { EDP } & \text { Sciences, } & \text { 2017, }\end{array}$

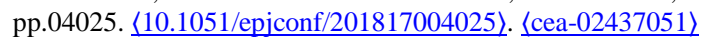

[9] E. Brun, F. Damian, C.M. Diop, E. Dumonteil, F.X. Hugot, C. Jouanne, Y.K. Lee, F. Malvagi, A. Mazzolo, O. Petit, J.C. Trama, T. Visonneau, A. Zoia, Tripoli-4®, CEA, EDF and AREVA reference Monte Carlo code, Annals of Nuclear Energy 82, 151-160 (2015).

[10] H. Carcreff et al., "Recent developments in nuclear heating measurement methods inside the OSIRIS reactor", Nuclear Instruments and Methods in Physics Research, A 942 (2019) 162310. 